\title{
Prenatal Diagnosis and Postnatal Management of a Large Hepatic Hemangioma
}

\author{
(1) Gürcan TÜRKYILMAZ, ${ }^{a}$ \\ (1) Emircan ERTÜRK, ${ }^{a}$ \\ (1) Tuğba SIVRIKOZ,a \\ (1) İbrahim KALELIOĞLU, ${ }^{a}$ \\ (1) Recep HAS, ${ }^{a}$ \\ (1) AtII YÜKSEL
}

\begin{abstract}
Hepatic hemangiomas are the most benign hepatic tumors appearing in childhood and the prenatal diagnosis is limited. An abdominal mass with a marked blood flow in prenatal USG examination is the major finding. Fetal MRI can be complementary to USG. While the majority of hepatic hemangiomas are asymptomatic, large lesions can lead to high-output cardiac failure, hydrops fetalis, anemia, and consumptive thrombocytopenia. Neonatal management is based on clinical findings and hematological parameters. We describe a case of hepatic hemangioma presenting a well-defined multicystic heterogeneous mass on obstetric ultrasound at the 22-week gestation which was managed conservatively in the postnatal period.
\end{abstract}

Keywords: Hemangioma, cavernous; liver neoplasms; magnetic resonance imaging; prenatal diagnosis; ultrasonography, Doppler, color

İstanbul Faculty of Medicine,

İstanbul

Received: 26.04 .2018

Received in revised form: 09.09.2018

Accepted: 10.09 .2018

Available online: 25.10 .2018

Correspondence:

Gürcan TÜRKYILMAZ

İstanbul University

İstanbul Faculty of Medicine,

Department of Obstetrics and Gynecology,

İstanbul, TURKEY

gurcanturkyilmaz@gmail.com

Copyright ( $~ 2018$ by Türkiye Klinikleri



epatic hemangiomas are benign tumors of vascular origin. They are estimated to constitute $1-5 \%$ of total pediatric malignancies and listed as most commonly diagnosed tumors after hepatic hepatoblastoma in pediatric patients. ${ }^{1,2}$ While some of these tumors may be unrecognized due to a small size in clinical practice, a noticeably large sized tumor may cause severe coagulopathy due to thrombocyte depletion and abdominal hemorrhage. This condition is called as Kasabach Merritt Syndrome and results from the rupture of the tumor. Prenatal di-agnosis is often based on the presence of an abdominal mass with a high blood flow appearing in color Doppler ultrasonography. Although small sized tumors can be managed expectantly, large sized tumors may require medical therapy (corticosteroids), surgical intervention, such as ligation of feeding arteries, mass excision or hepatic transplantation. We present prenatal diagnosis and postnatal management of a case with a hepatic hemangioma.

\section{CASE REPORT}

A gravida 2, para 0 woman, aged 28 years, approached our hospital due to intra abdominal cystic mass. There was no known risk factor in the obstetric history and first-trimester ultra-sound examination was normal. A detailed scan at 19 -week revealed a $22-\mathrm{mm}$ diameter heter-ogeneous, predominantly multicystic lesion in the upper right abdomen (Figure 1). 
An immensely vascular tumor was detected during the color and special Doppler examination (Figure 2). Gallbladder was seen in anatomic position. The biometry of the fetus was as per the gestational age. The amniotic fluid was within the normal range and placental localization was standard. No other fetal abnormality was detected and there was no evidence of fetal hydrops. Fetal heart examination revealed normal cardiac function and there was no cardio-megaly or tricuspid regurgitation. Fetal hepatic cavernous hemangioma was suspected. Fetal MRI was offered but the patient did not accept this intervention. Maternal AFP level was average.
Fetal ultrasound examination was repeated at 22 weeks of gestation. There was a slight in-crease in the tumor size and the fetal growth was compatible with gestational age. Arterial and venous Doppler evaluation revealed normal fetal wellbeing. The fetal examination was per-formed twice a month. Mass features remained stable and fetal color Doppler parameters were stable.

At 39 weeks of gestation, 3670 gr male infant was delivered spontaneously with Ap-gar scores 7 and 8 . The postnatal course was uncomplicated and abdominal examination was normal. The newborn was mildly anemic ( $\mathrm{Hb}: 9.7 \mathrm{~g} / \mathrm{dL})$, thrombocyte count, liver enzymes, coagulation parameters, and
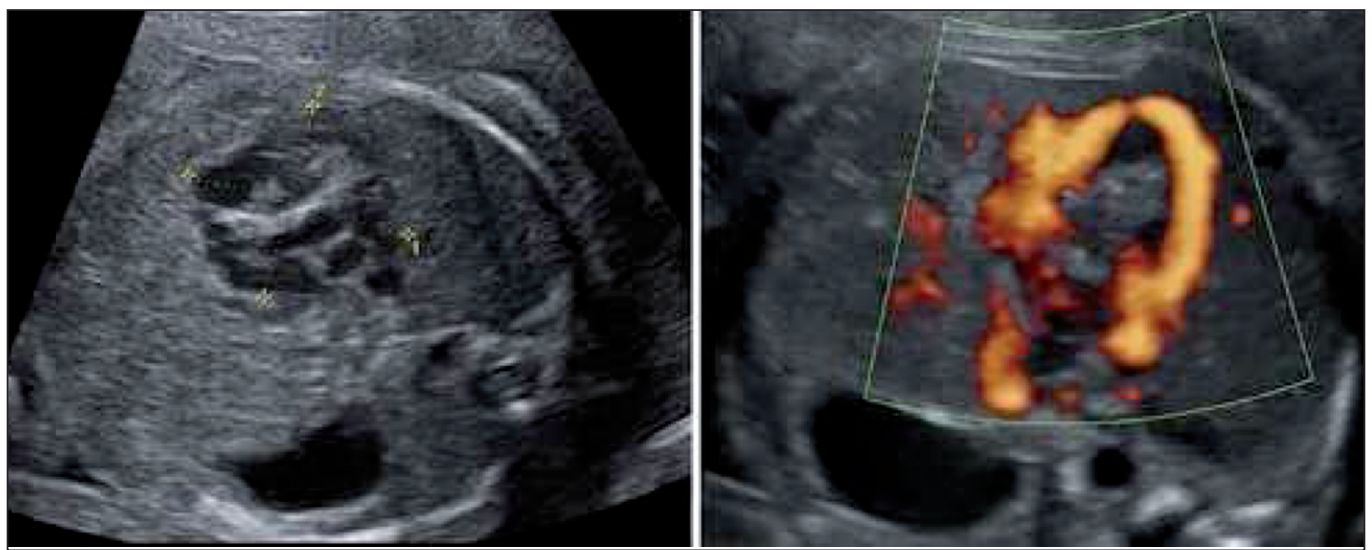

FIGURE 1: An axial ultrasound plan demonstrates the solid-cystic heterogeneous abdominal mass, and color Doppler image shows marked vascular nature of the tumor at 22 weeks of gestation.

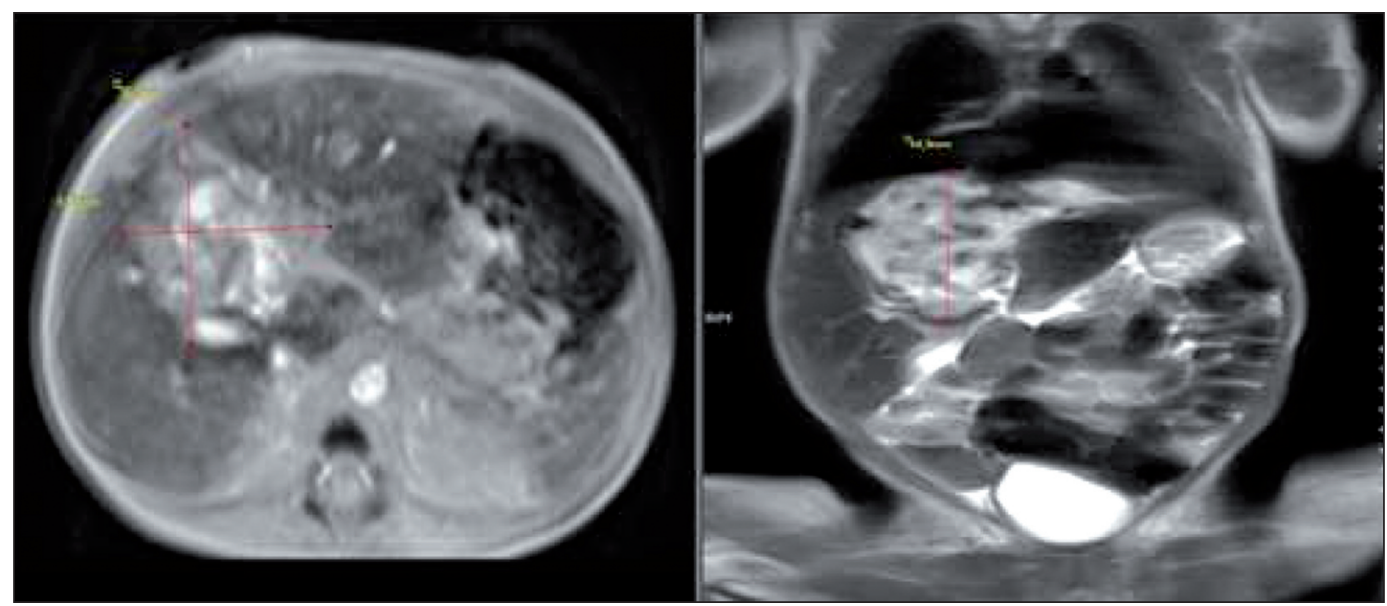

FIGURE 2: T2-weighted coronal and axial magnetic resonance images of the large hepatic hemangioma which shows contrast enhancement after gadolinium administration at one week of age. 
liver function tests were within normal range. During the ab-dominal ultrasound examination performed on postnatal day 1 , a markedly vascular, hetero-geneous, a multicystic mass was detected within the right hepatic lobe. A week later, a contrast-enhancing mass with a size of $42 \times 44 \times 40 \mathrm{~mm}$, showing low signal density on $\mathrm{T} 1$ weighted images and high signal intensity on T2 weighted images, was detected, confirming a diagnosis of liver hemangioma (Figure 2).

The laboratory parameters were in a normal range, thus the possibility of malignancy was ruled out. Steroid therapy was initiated and the patient was checked with ultrasound at the $6^{\text {th }}$ and $12^{\text {th }}$ months. No thrombocytopenia developed and a gradual decrease in the size of the tumor was observed during this period.

\section{DISCUSSION}

Although the rate of prenatal diagnosis of congenital tumors has increased gradually in recent years, most of these tumors are recognized at birth. The liver originated tumors are mostly detected during the perinatal period. Among these, hemangiomas, a mass consisted of endo-thelial-lined vascular structures, are the most common tumors during the neonatal period and childhood. They may appear as a focal solitary or multifocal lesion. Small hemangiomas are asymptomatic but rarely they may enlarge rapidly and cause serious complications.

The image of a hemangioma is distinct in ultrasound and can be characterized as a combination of cystic and solid areas in the upper abdomen. Punctate calcifications accompany $50 \%$ of cases. ${ }^{3}$ Color Doppler imaging is essential for differential diagnosis. Hemangiomas demonstrate hypervascularized, high blood flow lesions but hamartomas are predominantly solid avascular masses. Nowadays, prenatal MRI is preferred as a first-line diagnostic tool. MRI is not affected by maternal factors such as obesity thus provides better resolution than USG, and its multiplanar imaging capacity provides more information about the effect of tu-mor on adjacent organs. ${ }^{4}$ In our case, a heterogeneous, highly vascularized complex mass in the upper abdomen was diagnosed at 22 weeks. Differential diagnosis included hepatoblastomas and hamartomas. Hamartomas are commonly multicystic, multilocular lesions and show avascular contrast compared to hemangiomas. ${ }^{5}$ On the contrary, hepatoblastomas are counted as the most common tumors in the neonatal period but their prenatal diagnosis rate is relatively low. They are usually large, heterogeneous solid tumors and have weak vascularity like hamartomas. ${ }^{6,7}$ Maternal AFP increases in 5\% cases of hepatic hemangiomas but in our case, the AFP level was normal. ${ }^{8}$

Hepatic hemangiomas have a biphasic course that consists of first proliferation and then dec-rements in the tumor size. Their clinical expressions are variable and range from asymptomatic to fetal death. The mortality rate due to hepatic hemangiomas is $30-80 \%{ }^{9}$

Due to the shunting course of the tumor, mild to severe anemia, thrombocytopenia, consump-tive coagulopathy (Kasabach Merritt Syndrome), cardiac failure, and even death may occur. ${ }^{10}$ Owing to a very few case series in the literature, there is no prognostic ultrasound find-ing, and no consensus has been reached about follow up protocol. In our case, there was no sign of fetal anemia, cardiac failure, or hydrops. We examined the fetus fortnightly and lesion remained stable without any complications. Hemangiomas are not associated with aneuploidies, so we did not perform an amniocentesis.

A hepatic biopsy is contraindicated due to the risk of massive bleeding, and diagnosis is most often based on radiological findings. ${ }^{11}$ Ultrasonography often demonstrates isoechogenic septated lesions with an increased blood flow. Contrast- enhanced CT is better than USG in demonstrating any hypervascular lesion. MRI is the excellent diagnostic tool to show features of the lesion and decide the best treatment strategy. ${ }^{12}$

The prenatal management depends on gestational age and fetal complications. If anemia is suspected, fetal cord sampling is suggested. Thrombocyte count should be considered mean-while because thrombocytopenia is unpredictable. Packed erythrocytes and platelet concen-trates may be 
transfused. The role of corticosteroids via maternal administration or umbilical cord injection is under question. ${ }^{13}$ In postnatal period asymptomatic lesions could be man-aged conservatively with US and MRI with a follow up to monitor the expected regression. Neonates with significant heart failure or hematological complications should be treated with corticosteroids. Corticosteroid treatment has a $50-80 \%$ success rate and is the first line therapy. ${ }^{14}$ Antiproliferative agents such as vincristine and beta-blockade agents have shown positive effects but data are not sufficient to draw any firm conclusion. Nonetheless, if neo-nate continues to be unstable, surgical intervention is required. Hepatic artery ligation or surgical resection of the tumor should be considered, although medical and surgical therapies and liver transplantation may be the last option. In our case, the neonate was asymptomatic and hematological parameters were normal, so we managed the postnatal period conser vatively with USG follow up. In conclusion, fetal hepatic hemangiomas are commonly asymptomatic, however, may present with serious clinical complications such as high output cardiac failure, hydrops fetalis, fetal anemia, and thrombocytopenia (Kasabach-Merritt syndrome). Therefore, an accurate prenatal diagnosis and follow up are essential. Postnatal surveillance and treat-ment are based on clinical findings and hematologic parameters.

\section{Source of Finance}

During this study, no financial or spiritual support was received neither from any pharmaceutical company that has a direct connection with the research subject, nor from a company that provides or produces medical instruments and materials which may negatively affect the evaluation process of this study.

\section{Conflict of Interest}

No conflicts of interest between the authors and / or family members of the scientific and medical committee members or members of the potential conflicts of interest, counseling, expertise, working conditions, share holding and similar situations in any firm.

\section{Authorship Contributions}

Idea/Concept: Gürcan Türkyılmaz; Design: Gürcan Türkyılmaz; Control/Supervısion: Recep Has, Atıl Yüksel; Data Collection and/or Processing: Emircan Ertürk; Analysis and/or Interpretation: Gürcan Türkyılmaz; Literature Review: Gürcan Türkyılmaz, Tuğba Sivrikoz; Writing the Article: Gürcan Türkyılmaz, Emircan Ertürk; Critical Review: İbrahim Kalelioğlu; References and Fundings: Tuğba Sivrikoz; Materials: Gürcan Türkyılmaz.

\section{REFERENCES}

1. Emre S, McKenna GJ. Liver tumors in children. Pediatr Transplant 2004;8(6):632-8.

2. Helmberger TK, Ros PR, Mergo PJ, Tomczak $\mathrm{R}$, Reiser MF. Pediatric liver neoplasms: a radiologic-pathologic correlation. Eur Radiol 1999;9(7):1339-47.

3. Fishman SJ, Mulliken JB. Hemangiomas and vascular malformations of infancy and childhood. Pediatr Clin North Am 1993;40(6):1177-200.

4. Heaton TE, Liechty KW. Postnatal management of prenatally diagnosed abdominal masses and anomalies. Prenat Diagn 2008;28(7):656-66.

5. Avni FE, Massez A, Cassart M. Tumors of the fetal body: a review. Pediatr Radiol 2009; 39(11):1147-57

6. Catanzarite V, Hilfiker M, Daneshmand S, Willert J. Prenatal diagnosis of fetal hepato- blastoma: a case report and review of the literature. J Ultrasound Med 2008;27(7):10958.

7. Al-Hussein HA, Graham EM, Tekes A, Huisman TA. Pre- and postnatal imaging of a congenital hepatoblastoma. Fetal Diagn Ther 2011;30;(2):157-9.

8. Makin E, Davenpont M. Fetal and neonatal liver tumors. Early Hum Dev 2010;86(10):63742.

9. Mueller BU, Mulliken JB. The infant with a vascular tumor. Semin Perinatol 1999;23(4):33240.

10. Franchi-Abella S, Gorincour G, Avni F, Guibaud L, Chevret L, Pariente D. Hepatic hemangioma-prenatal imaging findings, complications and perinatal outcome in a case series. Pediatr Radiol 2012;42(3):298-307.
11. Kassarjian A, Zurakowski D, Dubois J, Paltiel HJ, Fishman SJ, Burrows PE. Infantile hepatic hemangiomas: clinical and imaging findings and their correlation with therapy. AJR Am J Roentgenol 2004;182(3):785-95

12. Pott Bärtsch EM, Paek BW, Yoshizawa J, Goldstein RB, Ferrell LD, Coakley FV, et al. Giant fetal hepatic hemangioma. Case report and literature review. Fetal Diagn Ther 2003;18(1):59-64.

13. Gembruch U, Baschat AA, Gloecjner-Hoffmann K, Gortner L, Germer U. Prenatal di-agnosis and management of fetuses with liver hemangiomata. Ultrasound Obstet Gynecol 2002;19(5):454-60

14. Aslan H, Dural O, Yildirim G, Acar DK. Prenatal diagnosis of a liver cavernous hemangioma. Fetal Pediatr Pathol 2013;32(5):341-5. 